\title{
Top-9-liste de la SSMI: faire plus avec moins
}

\author{
Thierry Fumeauxa ${ }^{a}$ Luca Lavinab
}

a Prof., président médecins SSMI, membre FMH; ${ }^{\text {b }}$ Secrétariat général SSMI

Au cours des derniers mois, plusieurs sociétés de discipline médicale ont présenté des listes d'interventions dont elles déconseillent la prescription en raison du trop faible bénéfice pour les patients ou même de leur caractère néfaste. Au mois de juin 2017, l'association de soutien Smarter Medicine a été fondée avec pour objectif de donner un nouvel élan à ces projets.

A une époque de hausse des frais de santé, les initiatives de ce genre sont essentielles, en particulier pour une discipline aussi sophistiquée que la médecine intensive. C'est la raison pour laquelle la Société Suisse de Médecine Intensive (SSMI) a lancé l'initiative Choosing Wisely et élaboré une liste de mesures dont la prescription dans les unités de soins intensifs suisses devrait être remise en question.

La médecine intensive focalise les défis de notre système de santé: des patients âgés ayant un grand besoin en soins se retrouvent au sein d'une discipline avec une grande densité de personnel et une vaste palette de technologies coûteuses et complexes, qui doivent toujours être disponibles. Le traitement d'un patient en unité de soins intensifs coûte deux à trois fois plus qu'un séjour hospitalier moyen [1].

\section{Interventions efficaces, scientifiquement prouvées}

C'est précisément parce que la médecine intensive représente une branche spécialisée de la médecine qui est très onéreuse et prend en charge des patients en état critique qu'il convient de n'ordonner que des interventions dont l'efficacité a été prouvée scientifiquement. En plus, les mesures prises ne devraient entraîner aucun effet indésirable significatif et ne devraient pas recouper une intervention déjà réalisée. Bien entendu, le traitement doit être effectivement nécessaire à la prise en charge du patient. Bien que ces critères soient pertinents pour la plupart des interventions réalisées dans les unités de soins intensifs du pays, des interventions pour lesquelles ces solides prérequis font défaut sont également réalisées: elles sont souvent prescrites par pure routine ou facilité.
La SSMI s'est donc chargée de déterminer comment atteindre de meilleurs résultats avec moins de moyens en médecine intensive et en soins intensifs. Ces efforts sont en parfait accord avec les objectifs de notre société interprofessionnelle. En effet, avec ses plus de mille membres, la SSMI s'engage fondamentalement pour une prise en charge efficace et de grande qualité de tous les patients en état critique.

\section{Groupe de travail interprofessionnel}

En 2016, la SSMI a mis en place un groupe de travail interprofessionnel et l'a chargé d'élaborer une liste regroupant les interventions de médecine intensive à remettre en question. Après l'évaluation minutieuse

En tant que société interprofessionnelle, nous nous engageons à évaluer le respect de la Top-9-liste au sein des unités de soins intensifs certifiées par la SSMI.

d'initiatives semblables prises par des sociétés de discipline médicale étrangères de médecine intensive, le groupe de travail en est arrivé à la conclusion qu'aucune des listes n'aurait pu être acceptée telle quelle. Afin de n'intégrer à la liste que des mesures pertinentes pour la médecine intensive et les soins intensifs en Suisse, qui par la suite soient bien acceptées au sein des unités de soins intensifs, le groupe de travail a dû adapter les listes existantes d'interventions de médecine intensive au contexte suisse. Le remaniement en profondeur et l'adaptation aux conditions générales à l'échelle nationale distingue de ce fait la liste de la SSMI de celles des autres sociétés nationales.

Afin de garantir que la liste de la SSMI ne contienne pas d'interventions qui ne sont généralement pas pratiquées en Suisse ou dont l'évitement serait difficilement réalisable, les membres de notre société ont été priés, lors de l'été 2016, d'évaluer la liste et de la comparer au quotidien de la médecine intensive. La plupart des participants à l'enquête étaient des médecins adjoints d'unités de soins intensifs avec au moins dix ans d'expérience professionnelle. Les hôpitaux universi- 
taires et non universitaires y étaient représentés à proportion quasi-égale.

Bien qu'il soit ressorti de l'enquête que de nombreuses mesures de notre liste sont déjà appliquées telles que le recommande la SSMI, celle-ci a dévoilé un certain potentiel d'amélioration. Les participants à l'étude se sont donc prononcés pour l'implémentation de l'ensemble des recommandations élaborées. Le recueil qui a suivi, publié sous le nom Top-9-liste, a été adopté à l'unanimité par l'assemblée générale de la SSMI en septembre 2016.

1. Limiter la sédation profonde des patients sous ventilation mécanique, par l'utilisation de niveaux de sédation évalués par des échelles validées, et en permettant un réveil journalier, même partiel

2. Limiter la transfusion de globules rouges chez les patients stables et ne saignant pas, avec un seuil transfusionnel de $70 \mathrm{~g} / \mathrm{l}$ d'hémoglobine

3. Ne pas administrer des antibiotiques à large spectre sans une évaluation initiale du caractère approprié du traitement, avec une évaluation quotidienne de la possibilité d'une désescalade

4. Ne pas poursuivre les manœuvres avancées de soutien de la vie chez les patients présentant un risque significatif de décès ou de séquelles sévères, sans avoir discuté au préalable avec le patient - ou ses proches qui le représentent - des buts thérapeutiques, en tenant compte des valeurs et des préférences personnelles du patient

5. Ne pas pratiquer des examens complémentaires de routine ou à intervalle régulier, mais uniquement dans le but de répondre à une question clinique spécifique pertinente pour le patient

6. Ne pas administrer de nutrition parentérale à un patient sans déficit nutritionnel dans les premiers quatre à six jours du séjour aux soins intensifs

7. Ne pas administrer de fluides intraveineux lors d'une insuffisance circulatoire sans une évaluation préalable de la réponse aux fluides basée sur un test dynamique systématiquement, mais seulement après évaluation de la balance risque/bénéfice, et en favorisant la nutrition entérale
9. Ne pas mettre en place des équipements invasifs (cathéters, sondes, drains) si aucun bénéfice n'est attendu pour le patient, et réévaluer la pertinence de leur maintien, dans le but d'une ablation la plus précoce possible

Contrairement à ce que certains médias ont rapporté, cette publication de la SSMI n'est pas une liste noire; nous tenions à le préciser à nouveau. La Top-9-liste n'est pas un index qui regroupe les interventions qui doivent absolument disparaître du catalogue de prestations ou de la médecine intensive suisse. L'accent est mis sur la réflexion. Nous souhaitons que notre liste conduise chaque membre de la SSMI à une critique de son propre travail, suscite des discussions entre les médecins, les soignants et soignantes et les patients, et naturellement assure une optimisation de la prise en charge des patients en état critique.

\section{L'engagement continue}

Pour la SSMI, l'engagement ne se résume absolument pas au lancement de sa propre liste. En tant que société interprofessionnelle, nous nous engageons à évaluer le respect de la Top-9-liste au sein des unités de soins intensifs certifiées par la SSMI. En outre, nous évalueront à l'avenir l'influence de la liste sur la qualité des traitements et procéderont si nécessaire à des adaptations au niveau des recommandations. Il sera du devoir de la Commission qualité de notre société d'accompagner l'implémentation de la liste et de vérifier constamment sa pertinence. La Commission qualité établira le lien entre la liste et les autres efforts de la SSMI dans le domaine de la gestion de la qualité, et s'assurera ainsi que notre liste participe de manière significative à une médecine intensive responsable et judicieuse. Enfin, comme l'a déclaré Martin Balmer (Aarau) lors du symposium SSMI de cette année, un travail de qualité n'apporte rien s'il ne s'avère pas, en fin de compte, bénéfique pour le patient.

\section{Référence \\ 1 Cooper et al. Medicare intensive care unit use: analysis of inci- dence, cost, and payment. Crit Care Med. 2004;32:2247-53.}

8. Ne pas administrer de prophylaxie antiulcéreuse
Vous pouvez télécharger et commander la brochure contenant la Top-9-liste de la SSMI à I'adresse suivante: http://sgi-ssmi.ch/ index.php/Outils_Promotionelles.html 